DR. EDUARDO LÓPEZ-CANEDA (Orcid ID : 0000-0003-1788-1556)

Article type : Original Research Article

\title{
The Think/No-Think Alcohol Task: A New Paradigm for Assessing Memory Suppression in Alcohol-Related Contexts
}

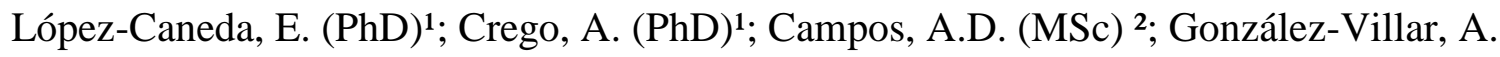 \\ $(\mathrm{PhD})^{3}$; Sampaio, A. $(\mathrm{PhD})^{1}$ \\ ${ }^{1}$ Psychological Neuroscience Lab, Research Center in Psychology (CIPsi), School of \\ Psychology, University of Minho, Braga, Portugal \\ ${ }^{2}$ Human Cognition Lab, Research Center in Psychology (CIPsi), School of Psychology, \\ University of Minho, Braga, Portugal \\ ${ }^{3}$ Department of Clinical Psychology and Psychobiology, University of Santiago de \\ Compostela, Galicia, Spain
}

\section{Corresponding author:}

Eduardo López-Caneda, Neuropsychophysiology Lab, Research Center in Psychology (CIPsi), School of Psychology, University of Minho, Campus Gualtar, 4710-057 Braga, Portugal.email: eduardo.lopez@psi.uminho.pt

\section{Acknowledgements}

This study was supported by the project NORTE-01-0145-FEDER-028672, funded by the Portuguese Foundation for Science and Technology (FCT) and the European Regional Development Fund (FEDER). Eduardo López-Caneda and Alberto Crego were supported by a Postdoctoral Fellowship of the FCT (SFRH/BPD/109750/2015 and

This article has been accepted for publication and undergone full peer review but has not been through the copyediting, typesetting, pagination and proofreading process, which may lead to differences between this version and the Version of Record. Please cite this article as doi: 10.1111/acer.13916

This article is protected by copyright. All rights reserved. 
SFRH/BPD/91440/2012, respectively), as well as by the Psychology Research Centre (UID/PSI/01662/2013), co-financed by FEDER through COMPETE2020 under the PT2020 Partnership Agreement (POCI-01-0145-FEDER-007653).

The authors have no conflicts of interest to disclose.

\begin{abstract}
Background: Research with the Think/No-Think (TNT) task has shown that voluntary suppression of an unwanted memory may lead to its later forgetting. To date, however, no study has assessed the memory suppression abilities in alcohol-related contexts despite the potential implications that it might have for alcohol research. With this aim, we developed a new version of the TNT paradigm, the Think/No-Think Alcohol (TNTA) task, which consists of 36 neutral pictures paired with 36 alcohol/no-alcohol images that are instructed to be suppressed or recollected. Methods: Electroencephalographic activity was recorded from 64 electrodes while 20 young healthy females performed the TNTA task. The event-related potentials (ERPs) typically involved in memory suppression/recollection were analyzed, namely the fronto-central N2, the late parietal positivity (LPP) and the frontal slow wave (FSW). Results: Findings revealed reduced recall for previously learned images that were subsequently instructed to be suppressed (No-Think) relative to those instructed to be retrieved (Think) and those not cued to be suppressed or retrieved (Baseline). This reduction seemed to be more prominent for alcohol-related memories. In addition, ERP analysis showed that compared to attempts of recollection, attempts of memory suppression were associated with attenuated LPP amplitude -more pronounced for alcohol-related memories(indicating reduced conscious recollection for No-Think images) as well as with increased FSW (suggesting strategic control aiming at decrease accessibility of unwanted memories). Conclusions: These results replicate and extend previously reported behavioral and ERP
\end{abstract}


findings in the TNT paradigm and suggest that the TNTA task may be a useful instrument to measure the ability to suppress alcohol-related memories.

Keywords: Memory Suppression, Recognition Memory, Think/No-Think, Event-Related Potentials, Alcohol Images

\section{Introduction}

Most individuals recall events they actually would prefer not to remember, including unpleasant or embarrassing memories from the past. What is less intuitive is the fact that some of these unwanted memories may indeed have been willingly removed from consciousness, thus promoting a subjective well-being (Nørby, 2015). Accordingly, several research lines suggest that people are able to voluntarily suppress memories of previously learned material (Depue, 2012; De Vito and Fenske, 2017; Hellerstedt et al., 2016; Lambert et al., 2010; Levy and Anderson, 2012; Waldhauser et al., 2012). In other words, some memories may become inaccessible as a result of several attempts of avoidance, a phenomenon known as suppression-induced forgetting or suppression of memories (Anderson and Hanslmayr, 2014; Banich and Depue, 2015; Murray et al., 2015).

One of the most used experimental paradigms to examine the intentional memory suppression is the Think/No-Think (TNT) task, a memory adaptation of the classical Go/NoGo task typically used to study suppression of motor responses (Huster et al., 2013). In this experimental approach, subjects are asked to learn cue-target pairs to a high degree of accuracy (Anderson and Green, 2001). Subsequently, only the cues are presented and they are instructed to either recall (Think condition) or suppress (No-Think condition) the other member of the pair (target). Through this task, numerous studies have found that memory for suppressed (No-Think) items is significantly reduced when compared to items instructed to 
be retrieved (Think items) and, most importantly, to items previously learned but not cued to be suppressed or recalled (Baseline items) (Benoit et al., 2015; Kim and Yi, 2013; Detre et al., 2013; Noreen and MacLeod, 2015). This mechanism of memory suppression has been related to increased activity in brain regions associated with inhibitory control, such as the inferior frontal gyrus or the dorsolateral prefrontal cortex, as well as to reduced activation in the hippocampus and sensory processing areas (Anderson et al., 2004; Depue et al., 2007; Gagnepain et al., 2014; Hulbert et al., 2016).

Event-related potential (ERPs) studies using the TNT paradigm have determined the presence of a number of ERP markers involved in attempts to suppress and/or retrieve memories. One of the most consistent findings is the reduction of the parietal memory effect for No-Think trials as compared with Think trials (Bergström et al., 2007; Cano and Knight, 2016; Chen et al., 2012; Depue et al., 2013). The parietal memory effect (also referred to as the old/new effect or the retrieval success effect) consists of a greater positivity for old (i.e., previously studied) items in comparison with new items (Doidge et al., 2017; Paller and Kutas, 1992; Rugg et al., 1996; Smith, 1993; Vilberg and Rugg, 2008). This positive wave, which arises approximately $400-800 \mathrm{~ms}$ after stimulus presentation, is largest over parietal scalp locations -often lateralized to the left hemisphere (Allan et al., 1998; Doidge et al., 2017)- and has been interpreted as an index of successful recollection (Friedman and Johnson, 2000; Rugg and Curran, 2007; Wilding and Ranganath, 2012; Yonelinas, 2002). During the TNT task, this late parietal positivity (LPP) is typically smaller for No-Think items than for Think items (Bergström et al., 2009b; Cano and Knight, 2016; Depue et al., 2013; Mecklinger et al., 2009; Zhang et al., 2016). The reduction in the LPP amplitude linked to the attempts of suppression is considered the ERP correlate of the attenuation of recollection-related activity in the hippocampal-parietal cortical network (Chen et al., 2012; Bergström et al., 2007; Mecklinger et al., 2009). 
Additionally, a negative deflection over the fronto-central region has been documented in several studies (Bergström et al., 2009a; Chen et al., 2012; Streb et al., 2016) when subjects try to suppress previously learned items. Both the spatial localization and time window ( 200-400 ms post-stimulus) has led some researchers to consider this component as functionally equivalent to the $\mathrm{N} 2$ waveform typically recorded in tasks involving cognitive control, such as the Go/NoGo task or the Stop signal task (Falkenstein, 2006; Huster et al., 2013). Thus, it is suggested that the fronto-central N2 component recorded during No-Think trials reflects the engagement of the prefrontal cortex (PFC) in the inhibition of the temporohippocampal activity, resulting in a gradual reduction of memory strength (Mecklinger et al., 2009; Streb et al., 2016).

One additional ERP finding reported from some studies using the TNT task is a more positive frontal slow wave (FSW) during memory suppression relative to memory retrieval (Bergström et al., 2007; Mecklinger et al., 2009; Waldhauser et al., 2012). This slow wave, usually arising between $600-900 \mathrm{~ms}$, is considered to be engaged in the strategic control of memory retrieval and, ultimately, in regulating (i.e., decreasing) the accessibility of unwanted memories in favor of intended recollections (Mecklinger, 2010; Waldhauser et al., 2012).

Recent research suggests that memory suppression may be impaired in several psychiatric conditions, such as posttraumatic stress disorder (Catarino et al., 2015), attention deficit hyperactivity disorder (Depue et al., 2010) or depressive disorders (Joorman et al., 2009). In the same vein, several studies point to abnormalities in memory control mechanisms of alcohol-dependent individuals as measured by the direct forgetting procedure (Noël et al., 2009; Todor, 2007) and the TNT task (Nemeth et al., 2014). Specifically, the Nemeth et al.'s study observed that, while alcohol-dependent patients did not differ from healthy controls in their baseline memory abilities, the instruction to inhibit retrieval did not lead to a significant 
decline of episodic recall in alcoholics, indicating that the capacity to suppress retrieval was impaired in these patients (Nemeth et al., 2014).

While it is known that it may be particularly difficult for individuals with alcohol abuse to refrain from thinking about alcohol when confronted by alcohol cues (Garland et al., 2012; Klein, 2007; Palfai et al., 1997), no study has yet used alcohol-related pictures within a TNT paradigm for assessing the ability to inhibit memories, probably because there is not yet a validated task examining the memory suppression abilities in alcohol-related contexts. Therefore, the purpose of the present study was to develop the Think/No-Think Alcohol (TNTA) task, a version of the TNT paradigm specifically designed for evaluating the individuals' ability to suppress alcohol and no-alcohol memories. We also aimed at testing whether the TNTA task was able to elicit -in young healthy subjects- the same behavioral and electrophysiological characteristics as such observed from the classical TNT task. Specifically, we hypothesized a below-baseline recall of No-Think items (i.e., memory for suppressed images would be reduced as compared to memory for baseline images) and that such a difference would be greater for alcohol-related pictures. Additionally, we expected that No-Think items would evoke an increased frontal N2 and an enhanced FSW as well as a reduced LPP in comparison with Think items.

\section{Material and Methods}

\section{Participants}

Thirty-six college and PhD students at the University of Minho (UM) were recruited for this study. All participants provided written informed consent prior to assessment. The exclusion criteria, assessed through a semi-structured interview, were as follows: noncorrected sensory deficits, history of traumatic brain injury or neurological disorder, personal history of psychopathological disorders (according to DSM-V criteria), consumption of 
medical drugs with psychoactive effects (e.g., sedatives or anxiolytics) during the week previous to the assessment, use of illegal drugs except cannabis as determined by the Drug Use Disorders Identification Test-Extended (DUDIT-E; Berman et al., 2007), scores $\geq 20$ in the Alcohol Use Disorder Identification Test (AUDIT; Babor et al., 2001) and a score above 90 in the Global Severity Index (GSI) of the Symptom Checklist-90 revised questionnaire (SCL-90-R; Degoratis, 1983) or in at least two of the symptomatic dimensions. A total of 25 participants (all of them females) who met the inclusion criteria were selected to perform the experiment. Five participants were excluded from posterior behavioral/EEG analysis: one of them because of extremely low performance in the learning phase of the TNTA task and the other four participants due to insufficient quality in the EEG signal, remaining a total of 20 participants (1 demographic and alcohol/other drugs consumption data are summarized in Table 1).

The study was approved by the Ethic Subcommission of Social and Human Sciences of the UM and the procedure was undertaken in accordance with the Code of Ethical Principles for Medical Research Involving Humans Subjects outlined in the Declaration of Helsinki (Brazil, 2013).

\section{Think/No-Think Alcohol Task}

Based on the classical TNT task and on further modifications (Depue et al, 2007), 36 alcohol and no-alcohol pictures obtained from the Galician Beverage Picture Set (GBPS; López-Caneda and Carbia, 2018) were paired with 36 images of neutral objects obtained from the POPORO database (Kovalenko et al., 2012).

The GBPS is a database of alcohol and no-alcohol pictures embedded in real-life scenarios which comprises six types of beverages: beer, wine and liquor (alcoholic drinks), and water, juice and milk (non-alcoholic drinks). The TNTA task included six images from 
each of the six beverages. The pictures also differed in terms of orientation (vertical or horizontal) and number of people (no people, one person, two or more people). As such, within each type of beverage, three were vertical (each one with a different number of people: 0 people, 1 person, 2 or more people) and the other three were horizontal (also with 0 people, 1 person, 2 or more people). The valence and arousal scores reported by the no/low drinkers (N/LDs) and the risky drinkers (RDs) in the study of López-Caneda and Carbia (2018) were taken into account for the images selection. Thus, those 18 alcohol-related images rated by RDs as more pleasant and arousing in comparison with N/LDs were selected for the alcohol pictures category. Furthermore, those no-alcohol pictures considered by N/LDs as being more pleasant and arousing in comparison with RDs were included within the no-alcohol pictures category.

On the other hand, for the selection of the 36 neutral objects images, 285 psychology undergraduate students rated -by mean of the Self-Assessment Manikin (Bradley and Lang, 1994)- 106 images of the POPORO database. The 36 pictures classified as more neutral those with values closer to 5 for both valence $(\mathrm{M}=4.8 \pm 0.5)$ and arousal $(\mathrm{M}=4.7 \pm 0.4)$ were extracted and randomly paired with the 36 alcohol/no-alcohol pictures.

The TNTA task was programmed in the open-source software Psychopy (Peirce, 2007) and included three phases: an initial learning phase, the subsequent Think/No-Think phase and a final memory test phase (see Figure 1).

\section{Learning Phase}

The learning phase consisted of the presentation of three series of 12 pairs (neutral object - alcoholic/non-alcoholic beverage). In each of the three series, participants were instructed to memorize all the object-beverage pairs, which were exposed for 4 seconds each at the center of a computer screen, in a randomized order and with an inter-stimuli interval of $1000 \mathrm{~ms}$ (with a rest of 4 seconds each 4 pairs). After each series of 12 pairs, participants 
were asked to recall -answering three questions- the associated target picture when being presented with the cue (neutral image). Questions were: Which beverage was associated with this picture? 1) water; 2) juice; 3) milk; 4) beer; 5) wine; 6) liquor"; "How was the picture oriented? 1) portrait; 2) landscape"; "How many people were there in the picture? 1) Nobody; 2) 1 person; 3) 2 or more people". Correct recall was only considered when participant provided the right answer to the three questions. Thus, the combination of the potential answers to the three questions $(2 \times 6 \times 3=36)$ ensured that each target image displayed a unique combination.

The three series of 12 pairs were presented at least twice and continued until participants were able to correctly recall the target pairs with at least $60 \%$ accuracy. The recall accuracy in this phase did not differ between alcohol and no-alcohol pictures (Mean accuracy $=76.9 \% \pm 9.6$ and $77.5 \% \pm 11.3$, respectively; $p=.86)$

\section{Think/No-Think Phase}

In this phase of the experiment, only the cue was presented. Participants were instructed to use the neutral picture as a memory cue to recall (Think trials) or to suppress (No-Think trials) the previously associated target picture. Therefore, 12 neutral object pictures served as Think trials, 12 as No-Think trials and the remaining 12 images were not presented during this phase and thus served as a behavioral baseline. All the pictures of this phase were presented for 4 seconds at the center of the screen (800-1200 ms inter-stimuli interval) and were repeated 10 times.

In the Think condition (neutral pictures with a green frame) participants were asked to "think of the previously learned picture and keep it in mind during the entire presentation". In the No-Think condition (neutral pictures with a red frame) they were instructed "not to let the previously associated picture enter your consciousness". They were also asked to keep fixation on the reminder stimulus and were discouraged from generating other associations to 
the reminder. The sequence of trials was pseudorandomized, with the restriction that the same condition did not occur more than 3 times in a row.

\section{Memory Test Phase}

During the memory test phase, all the 36 neutral pictures were presented, including the 12 pictures of the baseline condition, which had not been presented in the Think/NoThink phase. Participants were asked to recall the target picture that was initially associated with the cue, by answering the same three questions of the learning phase.

Three different versions of the task (where all the pictures were part of the three conditions: Think, No-Think and baseline) were created and counterbalanced across participants.

\section{EEG Acquisition}

Participants were asked to refrain from consuming alcohol at least $24 \mathrm{~h}$ before the EEG session. Additionally, they were instructed not to smoke, drink tea or coffee for at least $3 \mathrm{~h}$ before the assessment.

Each subject was seated in a comfortable armchair located in a light- and soundattenuated electrically shielded room. The EEG was recorded using the ActiveTwo Biosemi electrode system (Biosemi, Inc.) from 64 electrodes organized according the 10/10 system and sampled at $512 \mathrm{~Hz}$. Vertical and horizontal electro-oculogram activity was recorded to control for eye movements and blinks. Two additional electrodes were placed on the mastoids bilaterally to provide the signal reference. The impedances of all electrodes were kept below $20 \mathrm{k} \Omega$ and the EEG signal was filtered on-line with a $0.01-100 \mathrm{~Hz}$ band pass filter.

\section{ERP Analysis}


For the Think/No-Think phase, data were processed with BrainVision Analyzer software (Version 2.1). The EEG signal was corrected for vertical and horizontal ocular artifacts by independent component analysis (ICA) and re-referenced to the average reference. It was then digitally filtered off-line with a $0.1-30 \mathrm{~Hz}$ band-pass filter (12 $\mathrm{dB} /$ octave) and segmented into epochs of $1600 \mathrm{~ms}$ (from -100 to $1500 \mathrm{~ms}$ after stimulus onset). Additionally, baseline correction was applied and epochs exceeding $\pm 80 \mu \mathrm{V}$ at any scalp electrode were rejected.

Only trials corresponding to originally learned items during the learning phase were considered. There were no significant differences in the number of trials between the previously learned Think $(78.90 \pm 18.64)$ and the previously learned No-Think $(83.75 \pm$ 15.19) cue-target pairs $(\mathrm{p}=.24)$. Additionally, these epochs were averaged separately according to the type of picture to be recalled or suppressed, thus obtaining four conditions: Alcohol Think, No-Alcohol Think, Alcohol No-Think and No-Alcohol No-Think. The number of trials did not differ significantly across these four conditions $(p=.69): 39.5 \pm 2.5$ (Alcohol Think), $39.3 \pm 2.6$ (No-Alcohol Think), $41.4 \pm 2.4$ (Alcohol No-Think), $42.6 \pm 2.1$ (No-Alcohol No-Think).

To quantify the ERP data, we calculated the mean amplitudes for each electrode in three time windows, namely $200-400 \mathrm{~ms}$ and $600-1000 \mathrm{~ms}$ for fronto-central locations and 400-700 ms for parietal locations. Selection of the time windows and scalp regions was based on previous findings described above and on visual inspection of the ERP waveforms. Specifically, this selection was intended to quantify the N2, FSW and LPP components, respectively. As such, with the aim of exploring the N2 and the FSW amplitudes, we extracted the ERP data from six electrodes placed at left (F3, FC3), midline (Fz, FCz) and right (F4, FC4) frontal regions. To study the LPP component, statistical analyses of the ERP 
data was based on the following scalp electrodes: left parietal (P3, PO3), midline parietal (Pz, $\mathrm{POz}$ ) and right parietal (P4, PO4).

A $2 \times 2 \times 3 \times 2$ analysis of variance (ANOVA) was conducted on the mean amplitude of each component separately. Factors were Condition (Think, No-Think), Content (Alcohol, No-Alcohol), Region (Left, Midline, Right) and Electrode (two electrodes). In all cases, where appropriate, degrees of freedom were corrected by the Greenhouse-Geisser estimate, and post-hoc paired comparisons were performed with the Bonferroni adjustment for multiple comparisons (alpha level $\leq 0.05)$.

\section{Results}

\section{Behavioral Results}

Items that had been previously learned in the learning phase and which were correctly recalled during the memory test phase were considered correct responses. Thus, percentage of correct responses (for Think, No-Think and Baseline items) was computed according to the following formula: $\left(\frac{\text { number of correctly recalled items }}{\text { number of previously learned items }}\right) \times 100$.

The behavioral results are summarized in Table 2 and plotted in Figure 2. Two-way ANOVA with the factors Condition (Baseline, No-Think) and Content (Alcohol, NoAlcohol) revealed the expected bellow-baseline recall effect: recollection of No-Think items $(61.2 \% \pm 20.8)$ was significantly reduced in comparison with Baseline items $(74.2 \% \pm 19.3$; $\left.\mathrm{F}(1,19)=6.16, \mathrm{p}=.023, \eta_{\mathrm{p}}^{2}=.24\right)$ after 10 trial repetitions, replicating the classical behavioral findings typically reported in the TNT paradigm (e.g., Anderson and Green, 2001; De Vito and Fenske, 2017; del Prete et al., 2015; Kim and Yi, 2013; Levy and Anderson, 2008; Noreen and MacLeod, 2013). No significant effects for Content $(\mathrm{F}(1,19)=.07, \mathrm{p}=.79)$ or Condition x Content $(F(1,19)=.65, \mathrm{p}=.43)$ interaction were observed. Similarly, recall in 
the Think condition $(74.4 \% \pm 17.8)$ was significantly increased when compared to the NoThink condition $\left[\mathrm{F}(1,19)=6.52, \mathrm{p}=.019, \eta_{\mathrm{p}}{ }^{2}=.26\right]$ but did not differ significantly from Baseline $[\mathrm{F}(1,19)=.16, \mathrm{p}=.90]$. Additionally, planned comparisons in the form of pairedsamples t-tests revealed lower recall for Alcohol No-Think items $(29.6 \% \pm 11.8)$ as compared to recall for No-Alcohol Think items $(39.2 \% \pm 9.1 ; \mathrm{t}(19)=3.25, \mathrm{p}=.004)$ and Alcohol Baseline items $(38.6 \% \pm 13.0 ; \mathrm{t}(19)=2.49, \mathrm{p}=.022)$

Items that were only partially learned during the learning phase (i.e., memory for only one or two of the three questions) were also analyzed and their results are reported in the Supporting Information section and summarized in Table S1.

\section{ERP Results}

The grand averages of ERPs for Think (Alcohol and No-Alcohol) and No-Think (Alcohol and No-Alcohol) conditions are shown in Figures 4 and 5. Analysis of the LPP component revealed a marginally larger amplitude in the Think condition as compared to the No-Think condition $\left[\mathrm{F}(1,19)=4.02, \mathrm{p}=.059, \eta_{\mathrm{p}}{ }^{2}=.17\right]$. The Content factor showed significant effects $\left[\mathrm{F}(1,19)=15.04, \mathrm{p}<.001, \eta_{\mathrm{p}}{ }^{2}=.50\right]$, with larger amplitudes for no-alcohol than for alcohol images. In addition, a significant interaction between Condition $\mathrm{x}$ Region was found $\left[\mathrm{F}(2,38)=4.89, \mathrm{p}=.013, \eta_{\mathrm{p}}{ }^{2}=.20\right]$, and post-hoc analysis showed that amplitudes were larger for the Think condition than for the No-Think condition but only in the left $\left[\mathrm{F}(1,19)=6.12, \mathrm{p}=.023, \eta_{\mathrm{p}}{ }^{2}=.24\right]$ and midline $\left[\mathrm{F}(1,19)=5.64, \mathrm{p}=.028, \eta_{\mathrm{p}}{ }^{2}=.23\right]$ parietal regions, replicating the left parietal effect classically observed during memory suppression in the TNT paradigm (e.g., Bergström et al., 2007, 2009b; Cano and Knight, 2016; Depue et al., 2013; Hanslmayr et al., 2009). There was also a significant interaction between Condition $\mathrm{x}$ Content $\mathrm{x}$ Region $\left[\mathrm{F}(2,38)=3.80, \mathrm{p}=.031, \eta_{\mathrm{p}}{ }^{2}=.17\right]$, with this interaction appearing to be driven by the increased LPP amplitude for no-alcohol pictures relative to alcohol pictures at midline $\left[F(1,19)=4.90, p=.039, \eta_{p}{ }^{2}=.20\right]$ and right $\left[F(1,19)=6.01, p=.024, \eta_{p}{ }^{2}=.24\right]$ 
parietal regions in the Think condition and at left $\left[\mathrm{F}(1,19)=5.80, \mathrm{p}=.026, \eta_{\mathrm{p}}{ }^{2}=.23\right]$ and midline $\left[\mathrm{F}(1,19)=4.91, \mathrm{p}=.039, \eta_{\mathrm{p}}{ }^{2}=.20\right]$ parietal locations in the No-Think condition.

Guided by the behavioral results and with the aim of directly examining the NoAlcohol Think and the Alcohol No-Think conditions, we conducted an ANOVA with Condition/Content (No-Alcohol Think, Alcohol No-Think), Region (Left, Midline, Right) and Electrode (two electrodes) as factors. The results showed a significant effect of Condition/Content $\left[\mathrm{F}(1,19)=10.23, \mathrm{p}=.005, \eta_{\mathrm{p}}{ }^{2}=.35\right]$; the amplitude of LPP for NoAlcohol Think condition was significantly larger than for the Alcohol No-Think condition.

Analysis of the N2 component showed significant effects for the Content factor $\left[\mathrm{F}(1,19)=37.90, \mathrm{p}<.001, \eta_{\mathrm{p}}{ }^{2}=.66\right]$, with increased $\mathrm{N} 2$ amplitude (i.e., $\mathrm{N} 2$ became more negative) for no-alcohol in comparison with alcohol images. The Region factor also showed significant effect $\left[\mathrm{F}(2,38)=5.70, \mathrm{p}=.007, \eta_{\mathrm{p}}{ }^{2}=.23\right]$, with further analysis revealing larger $\mathrm{N} 2$ amplitude in the midfrontal regions than left frontal regions $\left[\mathrm{F}(2,18)=8.24, \mathrm{p}=.003, \eta_{\mathrm{p}}{ }^{2}\right.$ $=.48]$. Comparison between Think and No-Think conditions did not show significant main effects or interactions.

Analysis of the FSW revealed a significant main effect of Condition $[\mathrm{F}(1,19)=15.03$, $\left.\mathrm{p}=.001, \eta_{\mathrm{p}}{ }^{2}=.44\right]$, with larger FSW negativity for the Think trials in comparison with NoThink trials, and Content $\left[\mathrm{F}(1,19)=7.62, \mathrm{p}=.012, \eta_{\mathrm{p}}{ }^{2}=.29\right]$, where no-alcohol images elicited larger FSW negativity than alcohol images. No significant interactions were found involving the Condition factor.

\section{Discussion}

This study is the first to examine the memory suppression capacity in alcohol-related contexts in healthy young subjects. Using a modified version of the TNT paradigm -the TNTA task-, we were able to replicate the main behavioral finding obtained from the original 
procedure: recall for items instructed to be suppressed was significantly diminished as compared to baseline items, with some evidence that this reduction was more pronounced for alcohol-related memories. ERP analysis further revealed that compared to attempts of recollection, attempts of memory suppression were associated with reduced LPP, primarily at left and midline parietal regions, and with augmented FSW, which also mirrored previous EEG studies focused on avoidance of unwanted memories (Bergström et al., 2007; Mecklinger et al., 2009; Waldhauser et al., 2012).

Voluntary forgetting of previously learned material is a widely documented phenomenon. As mentioned above, research has shown that only a few attempts of suppression (between 5 and 10) are sufficient to impair memory of items learned a short time before (see Anderson and Hanslmayr, 2014; Banich and Depue, 2015 for recent reviews). This ability seems to further improve throughout childhood (Paz-Alonso et al., 2009) and to diminish in older adulthood (Anderson et al., 2011; Murray et al., 2015) and it has been reported from different modalities -visual and auditory (e.g., Cano and Knight, 2016; Gagnepain et al., 2017)- and using various types of stimuli, such as words, images or even autobiographical reminiscences (De Vito and Fenske, 2017; Depue et al., 2006; Hanslmayr et al., 2010; Noreen and MacLeod, 2013; Stephens et al., 2013).

Since the prominent work by Depue and cols. (2006, 2007), several TNT studies have used images as to-be-forgotten targets and most of them have replicated the findings initially obtained with verbal material (e.g., Benoit et al., 2016; Detre et al., 2013; Gagnepain et al., 2014). However, to date no study had used images of alcoholic and non-alcoholic beverages to examine the memory suppression abilities. Thus, this is the first study with alcohol-related material that replicates the results reported by Anderson and Green (2001). Specifically, we noted that No-Think instructions gave rise to lower recollection in the memory test phase than Think instructions and, most critically, than those items that were neither retrieved nor 
suppressed and thus acted as a behavioral baseline. Furthermore, we observed that memory impairment in the TNTA task was more prominent for items associated with alcohol images than for items associated with no-alcohol images, which seems to suggest that young healthy subjects with low to no alcohol use are more prone to forget alcohol-related material.

Alcohol images selected for this study were rated as being less pleasant and arousing by a similar sample of healthy college students with low or no alcohol use when compared to age-matched risky drinkers (López-Caneda and Carbia, 2018). Thus, due to its low emotional salience, it is likely that suppression of alcohol-related memories might have been intrinsically easier because these memories were less encoded, consolidated and, ultimately, retained than were no-alcohol ones. This result seems to fall in line with literature showing recollection impairment (i.e., augmented suppression) for low valence/unpleasant items compared to neutral or positive items (Depue et al., 2006; Joorman et al., 2005; Lambert et al., 2010; but see van Schie et al., 2013). Future studies should clarify if the opposite is true for subjects with high levels of alcohol consumption (e.g., binge drinkers or alcoholdependent individuals), namely, whether images with alcohol content are harder to voluntarily eliminate from consciousness.

The electrophysiological results also revealed some of the ERP correlates typically associated with the suppression and recollection processes involved in the TNT task. First, we found that the amplitude of the LPP over left and midline parietal regions was reduced for No-Think items as compared to Think items. This parietal positivity is considered to reflect the cortical activity supporting the representation of recollected information, i.e., the lower the amplitude, the lower the amount or quality of information retrieved (Vilberg et al., 2006; Wilding and Ranganath, 2012). Thus, the lower LPP amplitude observed for No-Think trials relative to Think trials may constitute an index of the reduction in the amount of conscious recollection. These findings converge with earlier ERP studies which have repeatedly 
reported a diminished parietal positivity in comparable time windows for No-Think relative to Think material (Bergström et al., 2007, 2009a; Cano and Knight, 2016; Chen et al., 2012; Depue et al., 2013; Mecklinger et al., 2009; Waldhauser et al., 2012; Zhang et al., 2016). In the same line, the lower LPP amplitude for suppressed alcohol-related memories than for recalled non-alcohol related memories might be indicative of a decrement in the amount of conscious recollection devoted to alcohol relative to no-alcohol material, which is also congruent with the behavioral evidence: Alcohol No-Think items displayed lower recollection rates than No-Alcohol Think items.

Regarding the N2 component, we failed to find significant differences between the Think and No-Think conditions. Hence, our results do not support the notion that the frontocentral N2 is involved in the avoidance of memory retrieval (Bergström et al., 2009a; Chen et al., 2012; Mecklinger et al., 2009). Rather, the similarities between N2 waveforms for both Think and No-Think conditions seem to indicate that this component would be more linked to familiarity-related recognition than to suppression of information. Accordingly, familiarity is often operationally defined as information that supports recognition in the absence of recollection (Mickes et al., 2009; Rugg and Curran, 2007) and it is suggested that its ERP correlate is a mid-frontal negative deflection peaking between $300-500 \mathrm{~ms}$ after stimulus onset -usually called the FN400 or mid-frontal old/new effect- (Curran and Hancock, 2007; Friedman and Johnson, 2000; Turk et al., 2018). The FN400, which topographically and temporally resembles the $\mathrm{N} 2$ component recorded in the present study, discriminates between non-studied (unfamiliar) versus studied (familiar) items (Curran and Cleary, 2003; Tsivilis et al., 2015; Woodruff et al., 2006). In the present study, it can be assumed that both types of items (Think and No-Think) were equally familiar, as both had been previously studied and learned, so the absence of differences in the $\mathrm{N} 2$ amplitude between these two conditions leads us to suggest that this component is functionally related to familiarity recognition rather than 
to retrieval suppression. However, further studies are necessary to determine whether the effect of familiarity may account for the convergent $\mathrm{N} 2$ findings observed in the Think and No-Think conditions.

On the other hand, the fact that $\mathrm{N} 2$ for alcohol and no-alcohol memories differed in amplitude might constitute an evidence for attentional differences that follow from viewing emotional content (Vuilleumier, 2005). Consistent with this idea, studies have generally found that, because of their motivational significance, emotionally salient stimuli elicit larger $\mathrm{N} 2$ amplitudes than neutral stimuli -though there are mixed reports as to whether this effect is equal for both pleasant and unpleasant stimuli- (Carretié et al., 2004; Hajcak et al., 2012; Olofsson et al., 2008). Thus, it is possible that the augmented N2 for non-alcoholic items (which were rated as more pleasant and arousing by N/LDs) is reflecting "attentional capture" or increase in the allocation of attentional resources toward non-alcohol related stimuli. Future studies directly comparing alcohol and no-alcohol memories between light and heavy drinkers might help to clarify the electrophysiological profile linked to this type of stimuli.

Finally, as predicted, to-be-suppressed items elicited more positive (although with slightly negative values) FSW relative to to-be-recalled items. Previous findings have also remarked that this slow and frontally focused component is increased during attempts of suppression when compared to efforts for recovering information (Bergström et al., 2007; Mecklinger et al., 2009; Waldhauser et al., 2012), which has been considered to reflect control mechanisms responsible for regulating competing memory traces in order to prevent unwanted memories (Mecklinger, 2010; Mecklinger et al., 2009; Waldhauser et al., 2012). Consistent with the literature, our results provide additional support in favor of this frontally mediated mechanism of control of unwanted memories in favor of intended recollections. 
The present study displays some limitations that deserve consideration. Firstly, the limited sample size could undermine the reliability of results, so additional research is needed to verify or refute the present findings. Secondly, because the sample consisted solely of females, the potential influence of gender was not explored in this study. Therefore, future studies should determine possible differences between both sexes. Finally, given that ovarian hormones may play an important role on brain function (Derntl et al., 2008; Goldstein et al., 2005), the lack of control on the menstrual cycle phase is another limitation of this study.

Despite these potential limitations, the present findings might shed new light on the understanding and treatment of alcohol use disorder. Alcohol craving -i.e., the strong subjective desire to drink- is recognized as a hallmark of alcohol dependence (American Psychiatric Association, 2013). As such, this psychiatric condition has been associated with increased reactivity to alcohol-related stimuli as well as with deficits in cognitive regulation of cue-induced craving (Heinz et al., 2009; Jasinska et al., 2014; Naqvi et al., 2015; Seo et al., 2013). The craving experience can be elicited by intrusive memories or thoughts about alcohol, which are often triggered by external cues such as alcohol billboards or social settings where alcohol is frequently consumed (Kavanagh et al., 2005; May et al., 2004; Verdejo-García and Bechara, 2009). Bearing this in mind, it can be suggested that an increase in the ability to exercise control over these thoughts could lead to a reduction in craving and, ultimately, in alcohol consumption. In this sense, previous studies have demonstrated that (motor) inhibition training can reduce alcohol intake (Houben et al., 2011, 2012; Jones and Field, 2013). Likewise, recent research has shown that memory suppression training enhances the ability to selectively forget unpleasant memories in both healthy subjects (Küpper et al., 2014) and depressed individuals (Joorman et al., 2009). However, the memory suppression abilities of alcoholics and heavy alcohol users as well as the potential of memory suppression 
training for reducing alcohol craving/drinking levels remain unexplored. Hopefully, development of the TNTA task may be a first step to fill this gap.

In conclusion, our results replicate and extend previous behavioral and ERP findings on the suppression-induced forgetting effect using a modified version of the TNT paradigm, the TNTA task. As such, the general finding described by Anderson and Green (2001) was also observed in the present study: a decline in the recall of No-Think items relative to the baseline condition, which seemed to be more prominent for alcohol-related material. In addition, mirroring ERP studies, attempts of memory suppression -relative to attempts of recollection- were associated with attenuated left parietal positivity -more pronounced for alcohol-related memories- (possibly indicating reduced conscious recollection) as well as with an increased slow frontal activity (suggesting strategic control on unwanted memories in order to decrease its accessibility). These findings may have significant implications as they clearly demonstrate that the TNTA task may be a useful instrument to measure the ability to suppress alcohol-related memories. Investigation concerning the extent to which heavy alcohol drinkers or individuals with alcohol abuse may have difficulties to suppress alcoholrelated material might entail important implications for both alcohol research and clinical purposes.

This article is protected by copyright. All rights reserved. 


\section{References}

Allan K, Wilding EL, Rugg MD (1998) Electrophysiological evidence for dissociable processes contributing to recollection. Acta Psychol 98:231-252.

American Psychiatric Association (2013) Diagnostic and Statistical Manual of Mental Disorders: DSM-5. 5th ed. American Psychiatric Association, Washington, DC.

Anderson MC, Green C (2001) Suppressing unwanted memories by executive control. Nature. 2001; 410:366-369.

Anderson MC, Hanslmayr S (2014) Neural mechanisms of motivated forgetting. Trends Cogn Sci 18:279-292.

Anderson MC, Ochsner KN, Kuhl B, Cooper J, Robertson E, Gabrieli SW, Glover GH, Gabrieli DE (2004) Neural systems underlying the suppression of unwanted memories. Science 303:232-235.

Anderson MC, Reinholz J, Kuhl BA, Mayr, U (2011) Intentional suppression of unwanted memories grows more difficult as we age. Psychol Aging 26:397-405.

Babor TF., Higgins-Biddle JC, Saunders JB, Monteiro MG (2001) AUDIT: The Alcohol Use Disorders Identification Test: Guidelines for Use in Primary Care. Department of Mental Health and Substance Dependence. World Health Organization, Geneva, Switzerland.

Banich MT, Depue BE (2015) Recent advances in understanding neural systems that support inhibitory control. Curr Opin Behav Sci 1:17-22.

Benoit RG, Davies DJ, Anderson MC (2016) Reducing future fears by suppressing the brain mechanisms underlying episodic simulation. Proc Natl Acad Sci U S A 113:E8492E8501.

This article is protected by copyright. All rights reserved. 
Benoit RG, Hulbert JC, Huddleston E, Anderson MC (2015) Adaptive top-down suppression of hippocampal activity and the purging of intrusive memories from consciousness. $\mathrm{J}$ Cogn Neurosci 27:96-111.

Bergström ZM, de Fockert JW, Richardson-Klavehn A (2009a) ERP and behavioural evidence for direct suppression of unwanted memories. NeuroImage 48:726-737.

Bergström ZM, de Fockert JW, Richardson-Klavehn A (2009b) Event-related potential evidence that automatic recollection can be voluntarily avoided. J Cogn Neurosci 21:1280-1301.

Bergström ZM, Velmans M, de Fockert J, Richardson-Klavehn A (2007) ERP evidence for successful voluntary avoidance of conscious recollection. Brain Res 1151:119-133.

Berman AH, Palmstierna T, Källmén H, Bergman H (2007) The self-report Drug Use Disorders Identification Test-Extended (DUDIT-E): reliability, validity, and motivational index. J Subst Abuse Treat 32:357-369.

Bradley MM, Lang PJ (1994) Measuring emotion: the self-assessment manikin and the semantic differential. J Behav Ther Exp Psychiatry 25:49-59.

Cano ME, Knight RT (2016) Behavioral and EEG Evidence for Auditory Memory Suppression. Front Hum Neurosci 10:133.

Carretié L, Hinojosa JA, Martín- Loeches M, Mercado F, Tapia M (2004) Automatic attention to emotional stimuli: neural correlates. Hum Brain Mapp 22:290-299.

Catarino A, Küpper CS, Werner-Seidler A, Dalgleish T, Anderson MC (2015) Failing to forget: Inhibitory-control deficits compromise memory suppression in posttraumatic stress disorder. Psychol Sci 26:604-616.

Chen C, Liu C, Huang R, Cheng D, Wu H, Xu P, Mai X, Luo Y-J (2012) Suppression of aversive memories associates with changes in early and late stages of neurocognitive processing. Neuropsychologia 50:2839-2848.

This article is protected by copyright. All rights reserved. 
Curran T, Cleary AM (2003) Using ERPs to dissociate recollection from familiarity in picture recognition. Cogn Brain Res 15:191-205.

Curran T, Hancock J (2007) The FN400 indexes familiarity-based recognition of faces. NeuroImage 36:464-471.

De Vito D, Fenske MJ (2017) Suppressing memories of words and familiar objects results in their affective devaluation: Evidence from Think/No-think tasks. Cognition 162:1-11.

del Prete, F, Hanczakowski M, Bajo MT, Mazzoni G (2015) Inhibitory effects of thought substitution in the think/no-think task: Evidence from independent cues. Memory 23:507-517.

Derntl B, Windischberger C, Robinson S, Lamplmayr E, Kryspin-Exner I, Gur RC, Moser E, Habel U (2008) Facial emotion recognition and amygdala activation are associated with menstrual cycle phase. Psychoneuroendocrinology 33:1031-1040.

Depue BE, Banich MT, Curran T (2006) Suppression of emotional and nonemotional content in memory: Effects of repetition on cognitive control. Psychol Sci 17:441-447.

Depue BE, Burgess GC, Willcutt EG, Ruzic L, Banich MT (2010) Inhibitory control of memory retrieval and motor processing associated with the right lateral prefrontal cortex: evidence from deficits in individuals with ADHD. Neuropsychologia 48:39093917.

Depue BE, Curran T, Banich MT (2007) Prefrontal regions orchestrate suppression of emotional memories via a two-phase process. Science 317:215-219.

Depue BE, Ketz N, Mollison MV, Nyhus E, Banich MT, Curran T (2013) ERPs and neural oscillations during volitional suppression of memory retrieval. J Cogn Neurosci 25:1624-1633.

Depue, BE (2012) A neuroanatomical model of prefrontal inhibitory modulation of memory retrieval. Neurosci Biobehav Rev 36:1382-1399.

This article is protected by copyright. All rights reserved. 
Derogatis LR (1983) Administration, Scoring and Procedures Manual II for the Revised Version of the SCL-90. Johns Hopkins University Press, Baltimore.

Detre GJ, Natarajan A, Gershman SJ, Norman KA (2013) Moderate levels of activation lead to forgetting in the think/no-think paradigm. Neuropsychologia 51:2371-2388.

Doidge AN, Evans LH, Herron JE, Wilding EL (2017) Separating content-specific retrieval from post-retrieval processing. Cortex 86:1-10.

Falkenstein M (2006) Inhibition, conflict and the Nogo-N2. Clin Neurophysiol 117:16381640.

Friedman D, Johnson Jr R (2000) Event- related potential (ERP) studies of memory encoding and retrieval: A selective review. Microsc Res Tech 51 6-28.

Gagnepain P, Henson, RN, Anderson MC (2014) Suppressing unwanted memories reduces their unconscious influence via targeted cortical inhibition. Proc Natl Acad Sci U S A 201311468.

Gagnepain P, Hulbert J, Anderson MC (2017) Parallel regulation of memory and emotion supports the suppression of intrusive memories. J Neurosci 2732-16.

Garland EL, Carter K, Ropes K, Howard MO (2012) Thought suppression, impaired regulation of urges, and Addiction-Stroop predict affect-modulated cue-reactivity among alcohol dependent adults. Biol Psychol 89: 87-93.

Goldstein JM, Jerram M, Poldrack R, Ahern T, Kennedy DN, Seidman LJ, Makris N (2005) Hormonal cycle modulates arousal circuitry in women using functional magnetic resonance imaging. J Neurosci 25:9309-9316.

Hajcak G, Weinberg A, MacNamara A, Foti D (2012) ERPs and the study of emotion, in The Oxford Handbook of Event-Related Potential Components (Luck SJ, Kappenman E eds), pp 441-474. Oxford University Press, Oxford.

This article is protected by copyright. All rights reserved. 
Hanslmayr S, Leipold P, Bäuml KH (2010) Anticipation boosts forgetting of voluntarily suppressed memories. Memory 18:252-257.

Hanslmayr S, Leipold P, Pastötter B, Bäuml KH (2009) Anticipatory signatures of voluntary memory suppression. J Neurosci 29:2742-2747.

Heinz A, Beck A, Grüsser SM, Grace AA, Wrase J (2009) Identifying the neural circuitry of alcohol craving and relapse vulnerability. Addict Biol 14:108-118.

Hellerstedt R, Johansson M, Anderson MC (2016) Tracking the intrusion of unwanted memories into awareness with event-related potentials. Neuropsychologia 89:510523.

Houben K, Havermans RC, Nederkoorn C, Jansen A (2012) Beer à No- Go: Learning to stop responding to alcohol cues reduces alcohol intake via reduced affective associations rather than increased response inhibition. Addiction 107:1280-1287.

Houben K, Nederkoorn C, Wiers RW, Jansen A (2011) Resisting temptation: decreasing alcohol-related affect and drinking behavior by training response inhibition. Drug Alcohol Depend 116:132-136.

Hulbert JC, Henson RN, Anderson MC (2016) Inducing amnesia through systemic suppression. Nat Commun 7:11003.

Huster RJ, Enriquez-Geppert S, Lavallee CF, Falkenstein M, Herrmann CS (2013) Electroencephalography of response inhibition tasks: functional networks and cognitive contributions. Int J Psychophysiol 87:217-233.

Jasinska AJ, Stein EA, Kaiser J, Naumer MJ, Yalachkov Y (2014) Factors modulating neural reactivity to drug cues in addiction: a survey of human neuroimaging studies. Neurosci Biobehav Rev 38:1-16.

Jones A, Field M (2013) The effects of cue-specific inhibition training on alcohol consumption in heavy social drinkers. Exp Clin Psychopharmacol 21:8-16.

This article is protected by copyright. All rights reserved. 
Joormann J, Hertel PT, Brozovich F, Gotlib IH (2005) Remembering the good, forgetting the bad: intentional forgetting of emotional material in depression. J Abnorm Psychol 114:640-648.

Joormann J, Hertel PT, LeMoult J, Gotlib IH (2009) Training forgetting of negative material in depression. J Abnorm Psychol 118:34-43.

Kavanagh DJ, Andrade J, May J (2005) Imaginary relish and exquisite torture: the elaborated intrusion theory of desire. Psychol Rev 112:446-467.

Kim K, Yi DJ (2013) Out of mind, out of sight: Perceptual consequences of memory suppression. Psychol Sci 24:569-574.

Klein AA (2007) Suppression-induced hyperaccessibility of thoughts in abstinent alcoholics: A preliminary investigation. Behav Res Ther 45:169-177.

Kovalenko LY, Chaumon M, Busch NA (2012) A pool of pairs of related objects (POPORO) for investigating visual semantic integration: behavioral and electrophysiological validation. Brain Topogr 25:272-284.

Küpper CS, Benoit RG, Dalgleish T, Anderson MC (2014) Direct suppression as a mechanism for controlling unpleasant memories in daily life. J Exp Psychol Gen 143:1443-1449.

Lambert AJ, Good KS, Kirk IJ (2010) Testing the repression hypothesis: Effects of emotional valence on memory suppression in the think-no think task. Conscious Cogn 19:281293.

Levy BJ, Anderson MC (2008) Individual differences in the suppression of unwanted memories: the executive deficit hypothesis. Acta Psychol 127:623-35.

Levy BJ, Anderson MC (2012) Purging of memories from conscious awareness tracked in the human brain. J Neurosci 32:16785-16794.

This article is protected by copyright. All rights reserved. 
López-Caneda E, Carbia C (2018) The Galician Beverage Picture Set (GBPS): A standardized database of alcohol and non-alcohol images. Drug Alcohol Depend 184:42-47.

May J, Andrade J, Panabokke N, Kavanagh D (2004) Images of desire: Cognitive models of craving. Memory 12:447-461.

Mecklinger A (2010) The control of long-term memory: brain systems and cognitive processes. Neurosci Biobehav Rev 34:1055-1065.

Mecklinger A, Parra M, Waldhauser GT (2009) ERP correlates of intentional forgetting. Brain Res 1255:132-147.

Mickes L, Wais PE, Wixted JT. Recollection is a continuous process: Implications for dualprocess theories of recognition memory. Psychol Sci. 2009; 20: 509-15.

Murray BD, Anderson MC, Kensinger EA (2015) Older adults can suppress unwanted memories when given an appropriate strategy. Psychol Aging 30:9-25.

Naqvi NH, Ochsner KN, Kober H, Kuerbis A, Feng T, Wall M, Morgenstern J (2015) Cognitive regulation of craving in alcohol- dependent and social drinkers. Alcohol Clin Exp Res 39:343-349.

Nemeth VL, Kurgyis E, Csifcsak G, Maraz A, Almasi DA, Drotos G, Szikszay P, Ando B, Janka Z, Must A (2014) The impact of intermediate-term alcohol abstinence on memory retrieval and suppression. Front Psychol 5:1396.

Noël X, Billieux J, Van der Linden M, Dan B, Hanak C, de Bournonville S, Baaurain C., Verbank P (2009) Impaired inhibition of proactive interference in abstinent individuals with alcoholism. J Clin Exp Neuropsychol 31:57-64.

Nørby S (2015) Why forget? On the adaptive value of memory loss. Perspectives on Psychol Sci 10:551-578.

This article is protected by copyright. All rights reserved. 
Noreen S, MacLeod MD (2013) It's all in the detail: Intentional forgetting of autobiographical memories using the autobiographical think/no-think task. J Exp Psychol Learn Mem Cogn 39:375-393.

Noreen S, MacLeod MD (2015) What do we really know about cognitive inhibition? Task demands and inhibitory effects across a range of memory and behavioural tasks. PloS ONE 10:e0134951.

Olofsson JK, Nordin S, Sequeira H, Polich J (2008) Affective picture processing: an integrative review of ERP findings. Biol Psychol 77:247-265.

Palfai TP, Monti PM, Colby SM, Rohsenow DJ (1997) Effects of suppressing the urge to drink on the accessibility of alcohol outcome expectancies. Behav Res Ther 35:59-65.

Paller KA, Kutas M (1992) Brain potentials during memory retrieval provide neurophysiological support for the distinction between conscious recollection and priming. J Cogn Neurosci 4:375-391.

Paz-Alonso PM, Ghetti S, Matlen BJ, Anderson MC, Bunge SA (2009) Memory suppression is an active process that improves over childhood. Front Hum Neurosci 3:24.

Peirce JW (2007) PsychoPy_Psychophysics software in Python. J Neurosci Methods 162:813.

Rugg MD, Curran T (2007) Event-related potentials and recognition memory. Trends Cogn Sci 11:251-257.

Rugg MD, Schloerscheidt AM, Doyle MC, Cox CJ, Patching GR (1996) Event-related potentials and the recollection of associative information. Cogn Brain Res 4:297-304.

Seo D, Lacadie CM, Tuit K, Hong KI, Constable RT, Sinha R (2013) Disrupted ventromedial prefrontal function, alcohol craving, and subsequent relapse risk. JAMA Psychiatry 70:727-739.

This article is protected by copyright. All rights reserved. 
Smith ME (1993) Neurophysiological manifestations of recollective experience during recognition memory judgments. J Cogn Neurosci 5:1-13.

Stephens E, Braid A, Hertel PT (2013) Suppression-induced reduction in the specificity of autobiographical memories. Clin Psychol Sci 1:163-169.

Streb M, Mecklinger A, Anderson MC, Lass-Hennemann J, Michael T (2016) Memory control ability modulates intrusive memories after analogue trauma. J Affect Disord 192:134-142.

Todor, I (2007) Memory distortions and anxiety in alcoholism: a directed-forgetting investigation. J Psychol 141:229-240.

Tsivilis D, Allan K, Roberts J, Williams N, Downes JJ, El-Deredy W (2015) Old-new ERP effects and remote memories: the late parietal effect is absent as recollection fails whereas the early mid-frontal effect persists as familiarity is retained. Front Hum Neurosci 9:532.

Turk KW, Elshaar AAA, Deason RG, Heyworth NC, Nagle C, Frustace B., Flannery S, Zumwalt A, Budson AE (2018) Late Positive Component Event-related Potential Amplitude Predicts Long-term Classroom-based Learning. J Cog Neurosci 30:13231329.

van Schie K, Geraerts E, Anderson, MC (2013) Emotional and non-emotional memories are suppressible under direct suppression instructions. Cogn Emot 27:1122-1131.

Verdejo-García A, Bechara A (2009) A somatic marker theory of addiction. Neuropharmacology 56:48-62.

Vilberg KL, Moosavi RF, Rugg MD (2006) The relationship between electrophysiological correlates of recollection and amount of information retrieved. Brain Res 1122:161170.

This article is protected by copyright. All rights reserved. 
Vilberg KL, Rugg MD (2008) Memory retrieval and the parietal cortex: a review of evidence from a dual-process perspective. Neuropsychologia 46:1787-1799.

Vuilleumier P (2005) How brains beware: neural mechanisms of emotional attention. Trends Cogn Sci 9:585-594.

Waldhauser GT, Lindgren M, Johansson M (2012) Intentional suppression can lead to a reduction of memory strength: behavioral and electrophysiological findings. Front Psychol 3:401.

Wilding EL, Ranganath C (2012) Electrophysiological correlates of episodic memory processes, in The Oxford Handbook of Event-Related Potential Components (Luck SJ, Kappenman E eds), pp 373-396. Oxford University Press, Oxford.

Woodruff CC, Hayama HR., Rugg MD (2006) Electrophysiological dissociation of the neural correlates of recollection and familiarity. Brain Res 1100: 125-135.

Yonelinas AP (2002) The nature of recollection and familiarity: A review of 30 years of research. J Mem Lang 46:441-517.

Zhang D, Xie H, Liu Y, Luo Y (2016) Neural correlates underlying impaired memory facilitation and suppression of negative material in depression. Sci Rep 6:37556.

This article is protected by copyright. All rights reserved. 


\section{Figure Legends}

Figure 1. Depiction of the Think/No-Think Alcohol task. During the learning phase, participants are asked to associate and memorize 36 object-beverage pairs. Subsequently, only the neutral object is presented and participants have to try to remember the target (alcohol/no-alcohol image) that was associated with the cue (neutral image). After verifying at least $60 \%$ successful recollection, they move on to the Think/No-Think phase. In the Think condition (neutral images with a green frame) participants are instructed to "think of the previously learned picture and keep it in mind during the entire presentation". In the NoThink condition (neutral images with a red frame) they are asked "not to let the previously associated picture enter your consciousness". All the images are presented for 4 seconds and repeated 10 times. During the memory test phase, the 36 neutral images are presented again, including the 12 pictures of the baseline condition which had not been presented in the TNT phase. Participants are asked to recall -answering the same three questions of the learning phase- the target image that was initially associated with the cue.

Figure 2. Bar plot showing recall accuracy for Think, No-Think and Baseline conditions. *p $\leq .05$

Figure 3. Bar plot showing recall accuracy for Alcohol Think, No-Alcohol Think, Alcohol Baseline, No-Alcohol Baseline, Alcohol No-Think and No-Alcohol No-Think conditions. *p $\leq .05$

This article is protected by copyright. All rights reserved. 
Figure 4. Grand average of event-related potentials for Think (green line) and No-Think (red line) conditions. Shaded in grey are depicted the three components analyzed: N2, frontal slow wave (FSW) and late parietal positivity (LPP). Averages are presented for Fz, F4, FCz, FC4, P3, Pz, PO3 and POz electrodes.

Figure 5. Grand average of event-related potentials for No-Alcohol Think (green line), Alcohol Think (dashed green line), No-Alcohol No-Think (red line) and Alcohol No-Think (dashed red line) trials. Shaded in grey are depicted the three components analyzed: N2, frontal slow wave (FSW) and late parietal positivity (LPP). Averages are presented for Fz, F4, FCz, FC4, P3, Pz, PO3 and POz electrodes.

\section{Table 1}

\begin{tabular}{cc}
\hline Age & $24.4 \pm 4.2$ \\
Handedness (right/left) & $20 / 0$ \\
Caucasian ethnicity (\%) & 100 \\
Regular use of cannabis ( $\geq$ once a week) & 0 \\
Use of illegal drugs (except cannabis) & 0 \\
Age of onset of regular drinking & $16.1 \pm 3.6$ \\
Drinks in a standard week & $2.2 \pm 3.0$ \\
Global Severity Index score (SCL-90-R) & $0.2 \pm 0.2$ \\
Total AUDIT score & $3.8 \pm 3.1$ \\
\hline
\end{tabular}

This article is protected by copyright. All rights reserved. 
Table 2

\begin{tabular}{cccc}
\hline & Alcohol Images & No-Alcohol Images & Total \\
\hline Think & $35.8 \pm 14.7$ & $39.2 \pm 10.1$ & $74.4 \pm 17.8$ \\
Baseline & $38.6 \pm 13.0$ & $35.6 \pm 13.8$ & $74.2 \pm 19.3$ \\
No-Think & $29.6 \pm 11.8$ & $31.5 \pm 14.0$ & $61.2 \pm 20.8$ \\
\hline
\end{tabular}

\section{Table Legend}

Table 1. Demographic and drinking characteristics of the sample (mean \pm SD).

Table 2. Recall accuracy (\%) for Think, Baseline and No-Think items (Total, Alcohol and No-Alcohol images).

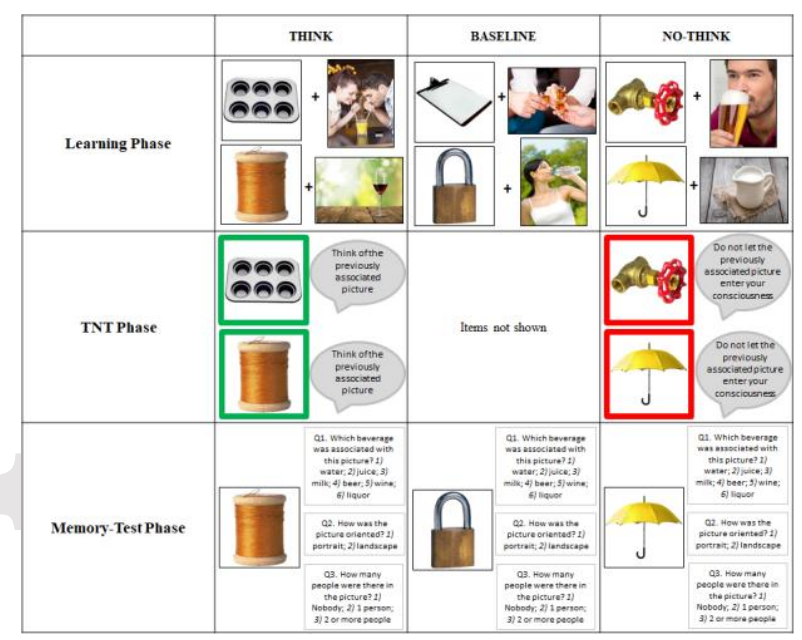

This article is protected by copyright. All rights reserved. 

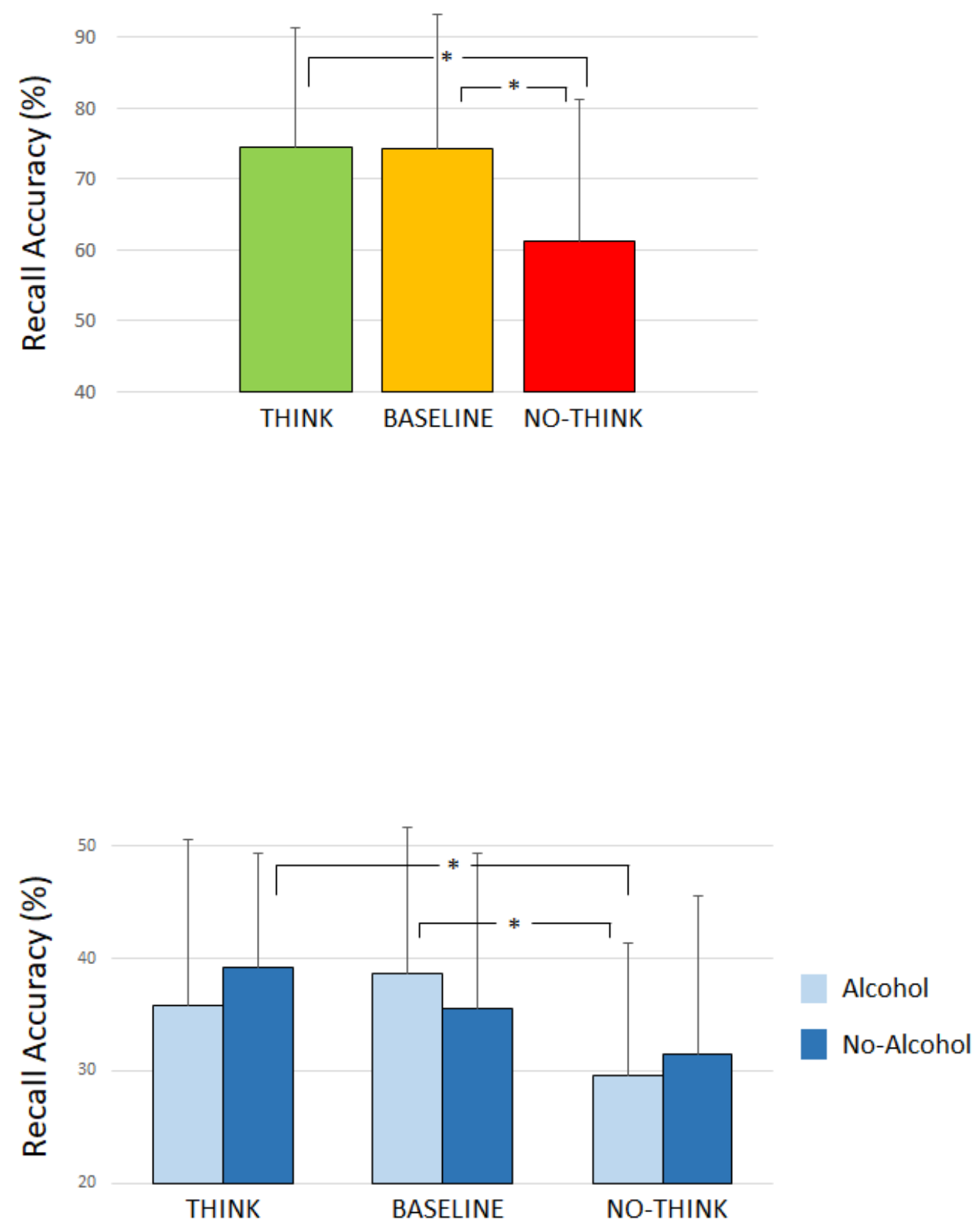

This article is protected by copyright. All rights reserved. 


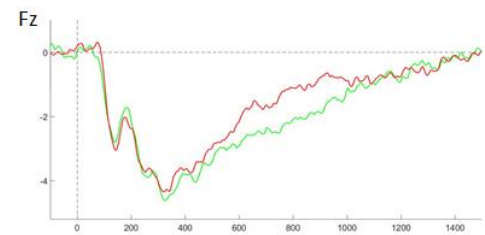

$\mathrm{FCz}$
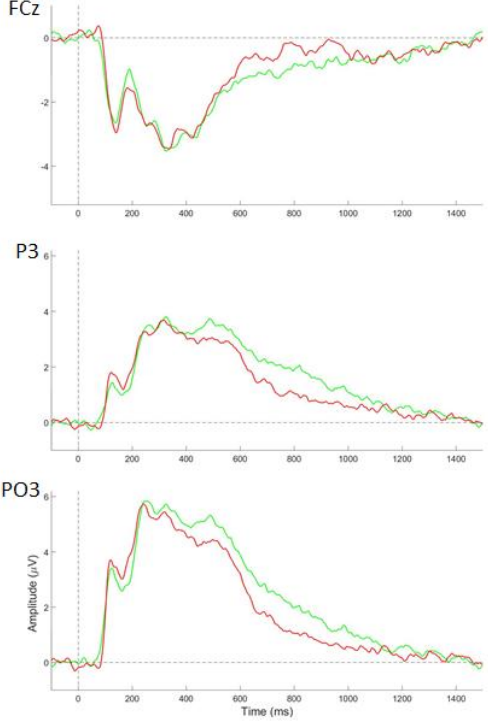

$\mathrm{Fz}$

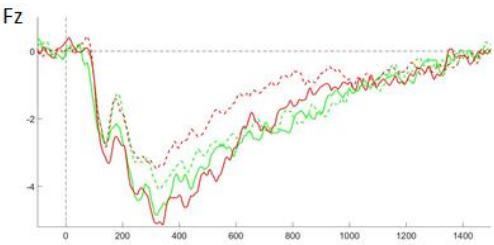

FCz
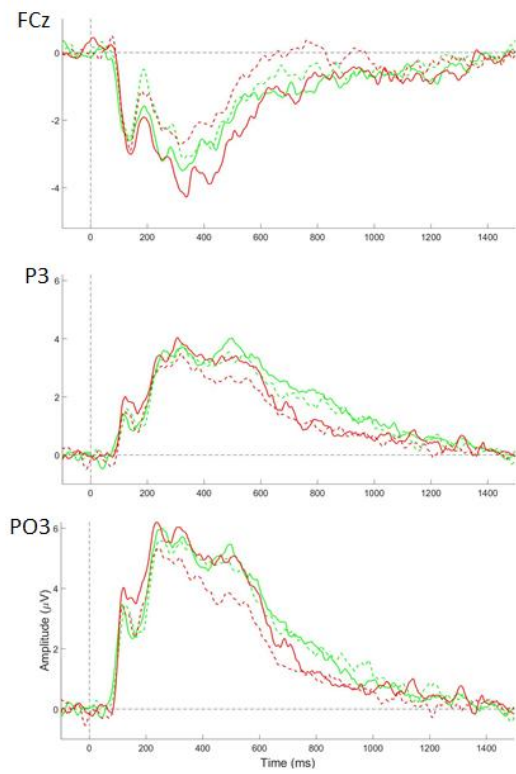

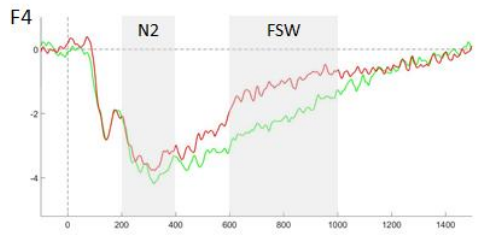

FC4
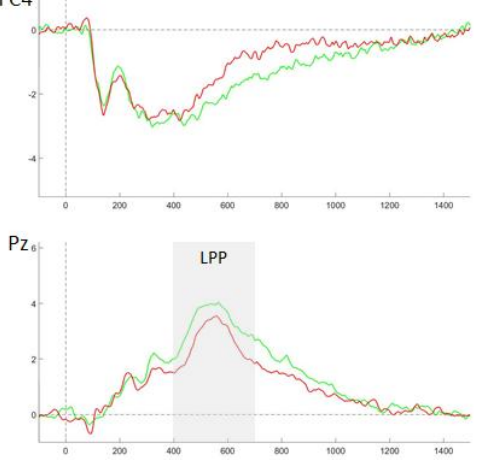

$\mathrm{POz}_{\text {。 }}$
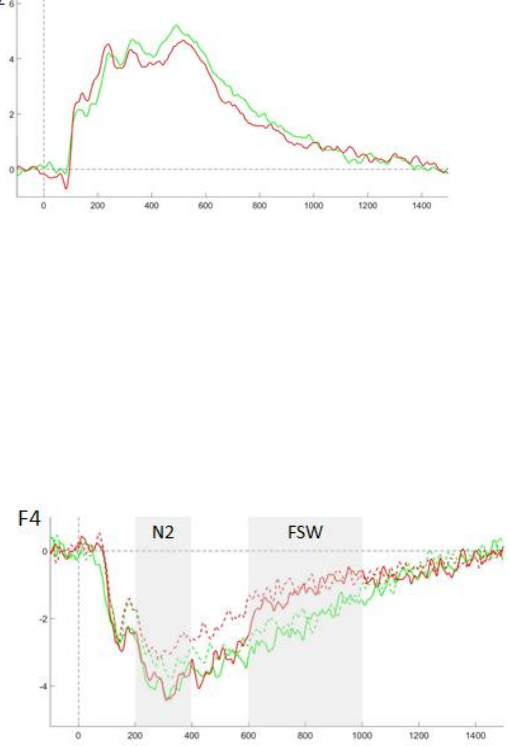

FC4
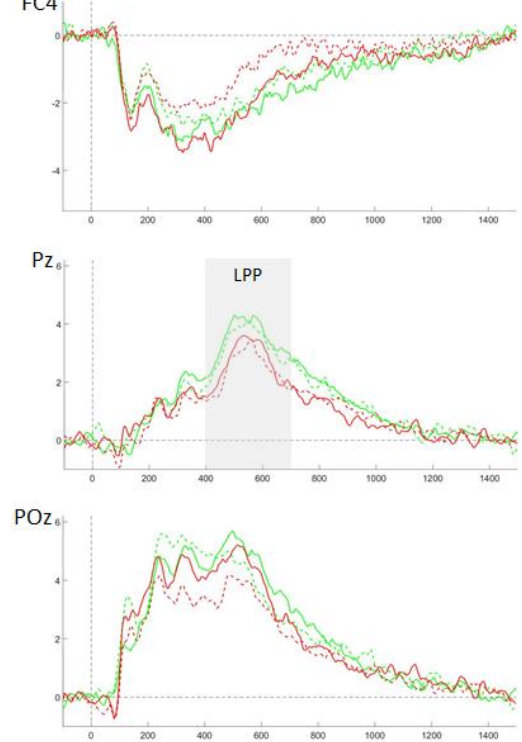

This article is protected by copyright. All rights reserved. 